\title{
A Tunable Scaffold of Microtubular Graphite for 3D Cell Growth
}

\author{
Constanze Lamprecht, ${ }^{*}{ }^{\dagger}, \#$ Mohammadreza Taale, ${ }^{\dagger}$ Ingo Paulowicz, ${ }^{\dagger}$ Hannes Westerhaus, ${ }^{\dagger}$ \\ Carsten Grabosch, ${ }^{\dagger}$ Arnim Schuchardt, ${ }^{\dagger}$ Matthias Mecklenburg, ${ }^{\ddagger}$ Martina Böttner, ${ }^{\S}$ Ralph Lucius, ${ }^{\S}$ \\ Karl Schulte, ${ }^{+}$Rainer Adelung, ${ }^{\dagger}$ and Christine Selhuber-Unkel ${ }^{\dagger}$ \\ ${ }^{\dagger}$ Institute for Materials Science, University of Kiel, 24143 Kiel, Germany \\ Institute of Polymers and Composites, Hamburg University of Technology, 21073 Hamburg, Germany \\ ${ }^{\S}$ Institute of Anatomy, University of Kiel, 24118 Kiel, Germany
}

Supporting Information

ABSTRACT: Aerographite (AG) is a novel carbon-based material that exists as a self-supportive $3 \mathrm{D}$ network of interconnected hollow microtubules. It can be synthesized in a variety of architectures tailored by the growth conditions. This flexibility in creating structures presents interesting bioengineering possibilities such as the generation of an artificial extracellular matrix. Here we have explored the feasibility and potential of AG as a scaffold for 3D cell growth employing cyclic RGD (cRGD) peptides coupled to poly(ethylene glycol) (PEG) conjugated phospholipids for surface functionalization to promote specific adhesion of fibroblast cells. Successful growth and invasion of the bulk material was followed over a period of 4 days.

KEYWORDS: aerographite, tissue engineering, 3D scaffold, cyclic RGD, fibroblasts

$\mathrm{D}$ eveloping novel materials for tissue regeneration requires the consideration of principles of engineering and life sciences. The natural extracellular matrix (ECM) provides a network of intricate collagen fibers that arrange in filaments of $2-20 \mu \mathrm{m}$ thickness to support cells, and guide their growth as well as their behavior. ${ }^{1}$ In order to imitate this topographical environment naturally derived and synthetic materials have been explored that are beginning to show notable progress. ${ }^{2-5}$ More recently, free-standing 3D scaffolds are increasingly favored over $2 \mathrm{D}$ materials to more accurately mimic the complex 3D cellular environment. ${ }^{4-7}$ Early work has shown promising results using microfiber constructs for organ reconstruction $^{8}$ and neuronal regeneration in animal models.

The macroscopic geometry is a key element in providing spatial organization for cell growth and appropriate nutritional conditions. Supplying oxygen and nutrients as well as waste removal by diffusion present growth constraints for cells in 3D. Thus, a conducive environment for cell growth and proliferation will be contingent on material porosity, pore size and interconnectivity of pores that allow cell migration and mass transport. The minimum pore size might be approximated by the diameter of cells in suspension, which depends on the cell type and varies broadly from 5 to $15 \mu \mathrm{m}$ for fibroblasts of connective tissue up to $100-350 \mu \mathrm{m}$ for bone. ${ }^{10}$

To mimic the ECM biochemically, cell-adhesive ligands, which are presented by the natural ECM in the form of fibronectin, vitronectin, and laminin, have to be included in the scaffold design. ${ }^{1}$ These ligands recruit cell surface receptors of the integrin-family, which play an active role in biochemical and mechanical signaling. ${ }^{11}$ A common motif of integrin-binding sites in fibronectin, vitronectin and laminin is the tripeptide
RGD of the L-amino acids arginin (R), glycine (G), and aspartic acid (D). It is widely used in synthetic materials to promote adhesion of a variety of cells. ${ }^{12-14}$

Ultralightweight aerographite $^{15,16}$ (AG) inherently fulfills the geometrical requirements posed by natural ECM very well. This novel material consists of a self-supporting highly porous (>99.9\% free volume) network of seamlessly interconnected hollow graphite tubes with micrometer-scale diameters and mechanical flexibility ( $\mathrm{kPa}$ modulus). Via surface functionalization, various biochemical signals may be introduced to provide appropriate conditions for $3 \mathrm{D}$ mammalian cell culture applications. In this study we investigated the use of AG as a scaffold for 3D cell growth for the first time. We employed cyclic RGD (cRGD) peptides coupled to poly(ethylene glycol) (PEG) conjugated phospholipids to promote specific adhesion of REF52 fibroblast cells and followed cell growth and invasion into the bulk material over a period of 4 days.

Conventional methods to fabricate $3 \mathrm{D}$ fibrous matrices include the decellularization of donor-derived matrices ${ }^{17}$ and electrospinning methods. ${ }^{18}$ AG synthesis, ${ }^{15}$ in contrast, is a one-step chemical vapor deposition (CVD) process. In brief, $\mathrm{ZnO}$ templates (Figure 1A, top panel) are produced from a loose powder of microsized $\mathrm{ZnO}$ tetra- and multipods that are compressed and heated for $3 \mathrm{~h}$ at $1200{ }^{\circ} \mathrm{C} .{ }^{19}$ Under an argon and hydrogen atmosphere with toluene as a carbon source the templates are converted into $\mathrm{AG}$ at $\sim 760{ }^{\circ} \mathrm{C}$. During

Received: January 20, 2016

Accepted: June 3, 2016

Published: June 3, 2016 

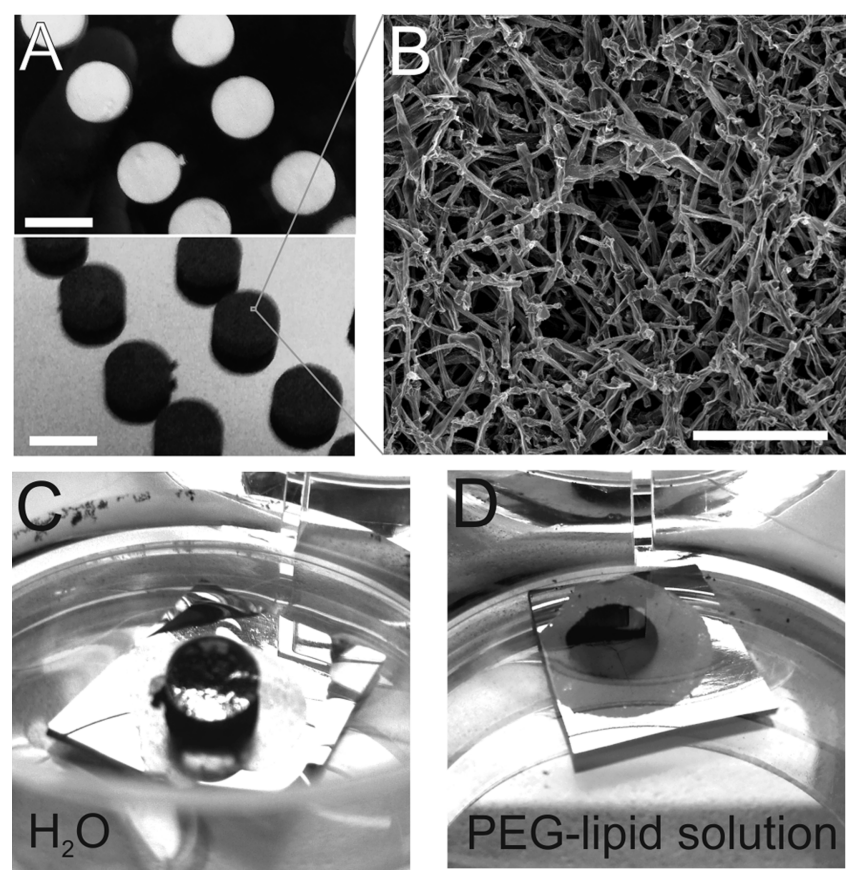

Figure 1. (A) White $\mathrm{ZnO}$ templates with a volume of $0.085 \mathrm{~cm}^{3}$ (top) are converted into black AG (bottom) in a one step CVD process. The $\mathrm{ZnO}$ is removed completely during formation of AG filaments. Scale bar: $6 \mathrm{~mm}$. (B) Scanning electron microscopy reveals the hierarchical scaffold of interconnected hollow carbon microtubules. Scale bar: 50 $\mu \mathrm{m}$. (C) AG is inherently super hydrophobic as demonstrated by water forming a nearly perfect droplet on the surface of the black AG disk, which is fixed to a small Si-chip with double-sided adhesive tape. (C) An aqueous solution of DSPE-PEG2000- $\mathrm{NH}_{2}$ is a well-suited wetting agent and the Si-chip with the AG disk readily submerges in the liquid.

deposition and formation of tubular graphitic carbon the underlying $\mathrm{ZnO}$ network is reduced to elemental $\mathrm{Zn}$ and removed entirely by the gas flow, resulting in a black opaque material (Figure 1A, bottom panel). An injection rate of 6 $\mathrm{mL} / \mathrm{h}$ per $\mathrm{g}(\mathrm{ZnO})$ yielded sample densities of $1.0-1.2 \mathrm{mg} / \mathrm{cm}^{3}$ and a Young's modulus of about $1 \mathrm{kPa}$.

Because the conversion process follows the template exactly, the resultant scaffold exhibits the same architecture and porosity. Hence, pore size and macroscopic shape of AG can be freely tailored through manipulating the template. By adjusting CVD parameters it is possible to tune filament diameter, thickness and aspect ratio and yield elastic moduli from $1 \mathrm{kPa}$ up to several $100 \mathrm{kPa}^{15}$, which allows for a multitude of bioengineering possibilities. ${ }^{20}$

AG scaffolds in this study exhibited pore sizes varying from $10 \mu \mathrm{m}$ to about $100 \mu \mathrm{m}$ and filaments with diameters between 0.5 and $3 \mu \mathrm{m}$ (Figure 1B), which compares well with natural ECM. ${ }^{1}$ Other carbon-based templates, such as graphenefoams ${ }^{21}$ or graphene oxide scaffolds ${ }^{22}$ lack the fibrous nature and form much larger pores. Capillary force induced restructuring of carbon nanotube-based networks, on the other hand, leads to confined cavities that are not accessible from all sides, ${ }^{23}$ whereas AG provides interconnected pores that afford penetrability and accessibility of all surfaces. In addition, the combination of ultralightweight and negligible volume fraction may prove advantageous for cells. After initial attachment to the scaffold, ECM producing cells may restructure and remodel their environment according to their adhesion needs through deposition of natural ECM. At the same time the hierarchical architecture of $A G$ is able to withstand strong deformations making AG networks mechanically flexible. $^{15}$

However, the application of AG in the biomedical field is initially hampered by its superhydrophobic nature (Figure 1C). To overcome this barrier, noncovalent functionalization schemes using amphiphilic molecules can be very attractive, as they do not require elaborate chemical modification that may alter the surface composition. Moreover, as pharmaceutical products often contain surfactants, a comprehensive library of approved agents is already available. In this study, we tested
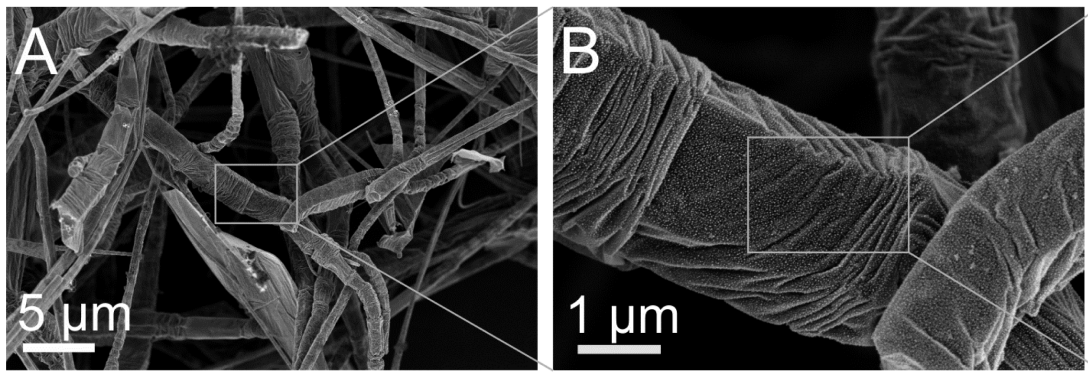

$\mathrm{D}$

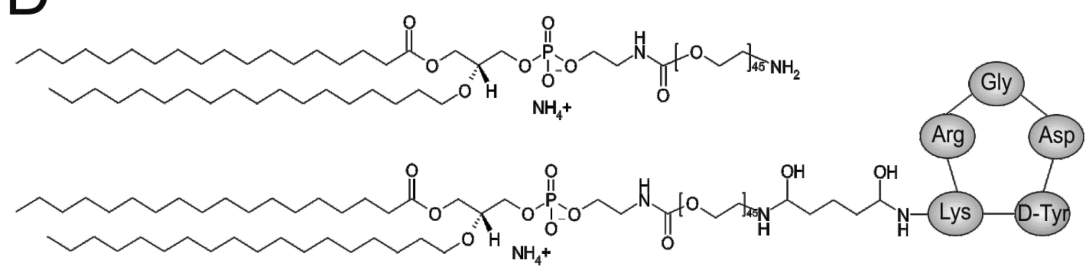

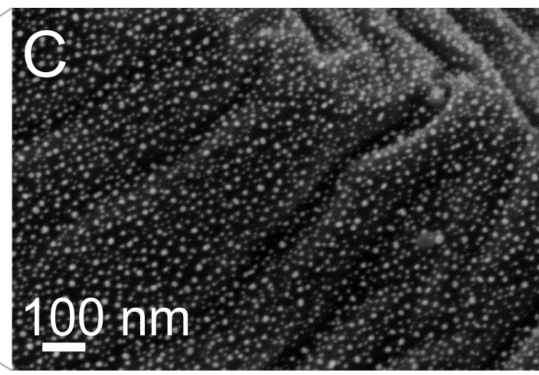

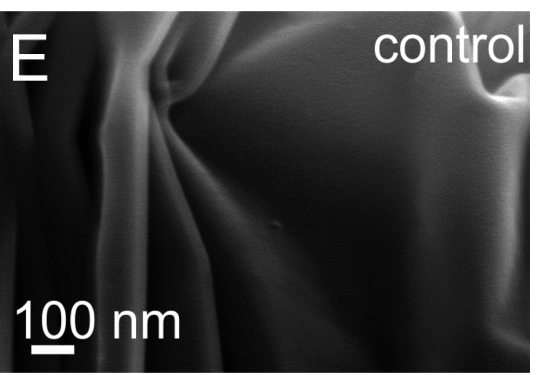

Figure 2. PEG-lipid functionalized AG was subjected to supercritical point drying followed by deposition of a thin layer of gold. (A-C) Gradual zoom-in reveals the adsorbed PEG-lipid molecules at high magnification. (D) A 4:1 mixture of amine terminated (top) and cyclic RGD peptide (cRGD) functionalized PEG-lipids (bottom) was used to promote cell attachment by integrin-mediated binding to cRGD. (E) Gold-coated pristine AG exhibits a smooth surface. 

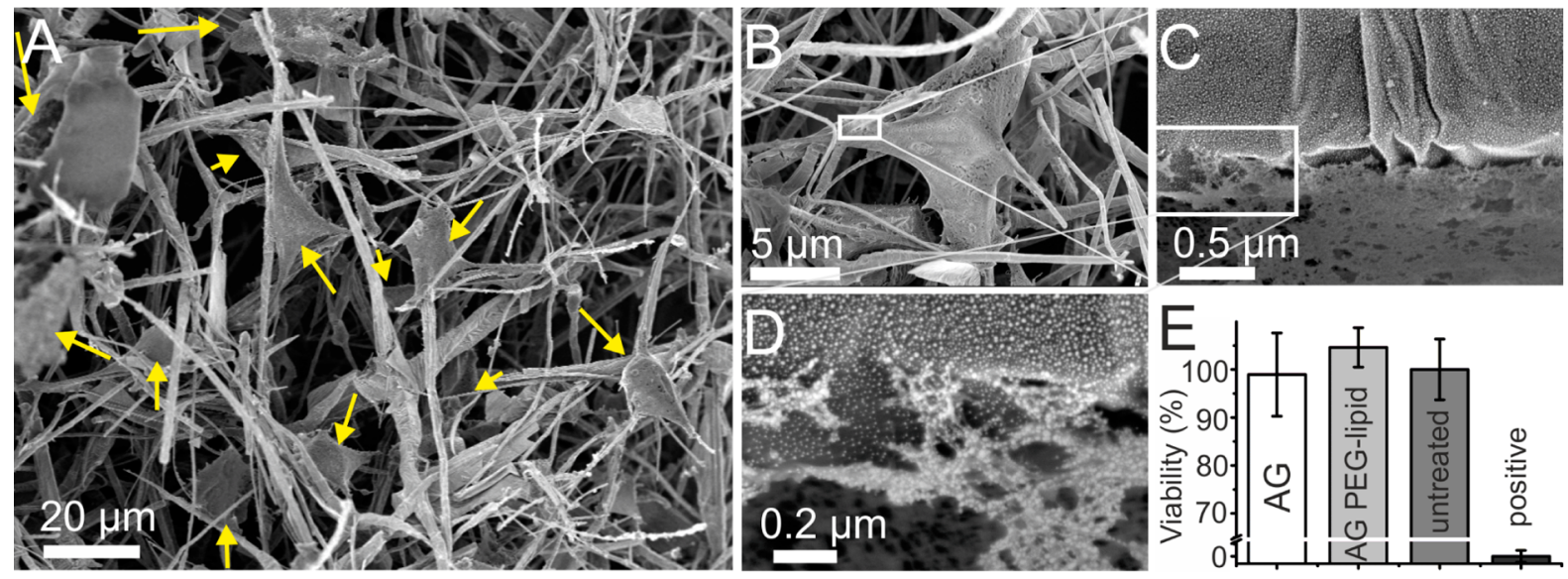

Figure 3. SEM images of REF 52 cells after 4 days of growth within cRGD functionalized AG with a 4:1 mixture of DSPE-PEG2000-NH $2 /$ DSPEPEG2000-cRGD. (A) The medium sized overview scan shows growth of numerous cells (arrows) along fibers in different planes within the 3D network. (B-D) Zoom-in on the interface between cell and functionalized AG surface show a tight physical connection between cells and scaffold material. (E) Results of MTT-Formazan absorbance measurement, showing mean values of cell viability (two independent experiments, three technical repeats in each of them) and \pm standard deviation for REF 52 cells treated with extracts of pristine (AG) and PEG-lipid conjugated (AG PEG-lipid) aerographite, as well as normal medium (untreated) and 15\% DMSO (positive).

different agents (Supporting Information) including lipidpoly(ethylene glycol) (PEG-lipid) to improve the immersion properties of AG. In that regard the open mostly unconstrained interconnected pore space in AG should facilitate wetting of all surfaces within the bulk of the material. Amine terminated PEG conjugated phospholipid (DSPE-PEG2000- $\mathrm{NH}_{2}$ ) yielded immediate and complete immersion of $\mathrm{AG}$ at a surfactant concentration of $1 \mathrm{mg} / \mathrm{mL}$ in distilled water (Figure 1C). PEGlipids are widely used in medical products ${ }^{24}$ and have been shown to successfully disperse various carbon based materials in aqueous media. ${ }^{25}$ The hydrophobic alkyl chains of the lipid part adsorbs onto the strongly hydrophobic surface of the carbon material, whereas PEG extends into the aqueous phase to impart hydrophilicity. ${ }^{24}$ Immersed samples were subjected to vacuum treatment to remove all air out from the bulk and ensure wetting of all surfaces.

Scanning electron microscopy (SEM) was performed to verify adsorption of PEG-lipids on the outer surface of the filaments (Figure 2). AG samples were dehydrated by super critical point drying (CPD) to avoid the destructive effect of surface tension on the network during evaporation of the liquid and a thin layer of gold was applied to improve visualization of biomolecules on the graphitic filaments. The PEG-lipids became visible at high magnification as bright dots (Figure $2 \mathrm{~B}, \mathrm{C})$, and were found to decorate the surface as a dense and homogeneous monolayer of individual molecules. Control experiments with gold-coated pristine AG confirmed that the observed nanostructures were not artifacts of the coating procedure (Figure 2E).

Amine terminated PEG-lipids offer several advantages: longchain PEG conveys inertness to the surfaces and prevents nonspecific binding of cells and proteins. The amine can be used for standard coupling of ligands, antibodies or therapeutic molecules to introduce specific functionalities, such as integrin mediated cell adhesion. Here we used cyclic RGD (cRGD) as ligand for the $\alpha_{v} \beta_{3}$ integrin in the plasma membrane of rat embryo fibroblasts (REF52). Fibroblasts were chosen in this pilot study, because this cell type synthesizes and deposits ECM to create an environment best suited to their function. ${ }^{7}$ AG scaffolds were functionalized with a 4:1 mixture of DSPEPEG2000-NH $\mathrm{NH}_{2}$ :DSPE-PEG2000-cRGD (Figure 2D). Assuming a uniform mixing of both types of PEG-lipids and taking into account a length of $9 \mathrm{~nm}$ for fully extended PEG2000, a ratio of 4:1 would yield a maximum spacing of integrin binding cRGDsites of $45 \mathrm{~nm}$. This is well within the range of distances that promote attachment and stable formation of focal adhesions by REF52 fibroblasts. ${ }^{26}$

REF52 were cultured for 4 days, then fixed with paraformaldehyde and prepared for SEM imaging by CPD. A thin layer of gold was applied for visualization of cells within the scaffolds and reduction of the destructive influence of the electron beam on biological samples. SEM images of fibroblasts near the surface of the AG bulk material (Figure 3) revealed the typical polygonal cell shape with elongated cytoplasm projections attaching to the scaffold. Images at higher magnification (Figure 3C,D) showed contact formation of the plasma membrane with the AG surface indicating the ability of cRGD functionalized AG to promote integrin mediated specific cell adhesion. The viability of cells upon exposure to pristine and DSPE-PEG2000- $\mathrm{NH}_{2}$ functionalized AG was tested according to the norm ISO 10993, which proposes standardized conditions for biological evaluation of medical devices and materials. In particular, assay protocols outlined in parts 5 (ISO 10993-5:2009) and 12 (ISO 10993-12:2004) of this norm were applied. Briefly, REF52 cells were cultured for $24 \mathrm{~h}$ in extract medium that had been incubated with AG and PEGlipid conjugated $\mathrm{AG}$ at $37{ }^{\circ} \mathrm{C}$ for $72 \mathrm{~h}$. To determine cell viability the colorimetric MTT metabolic activity assay was used with cells incubated in untreated medium as negative control and cells incubated in $15 \%$ DMSO as positive control (Figure 3E). The results were normalized to the viability of the negative control and show neither a negative effect of functionalized AG nor pristine AG on REF 52 cells.

Next, we explored the colonization depth within AG scaffolds using inherently fluorescent REF52 cells, which express YFPpaxillin. Paxillin is mainly located in the focal contacts formed by fibroblast upon adhesion (Figure 4A). Despite the extreme low density and open porous structure, AG scaffolds are opaque and have tremendous light absorbing capacities. ${ }^{15}$ Thus, fluorescence imaging proved most challenging and required extended illumination times of up to $5 \mathrm{~s} /$ frame. Optical image stacks were recorded up to a maximum penetration depth of 

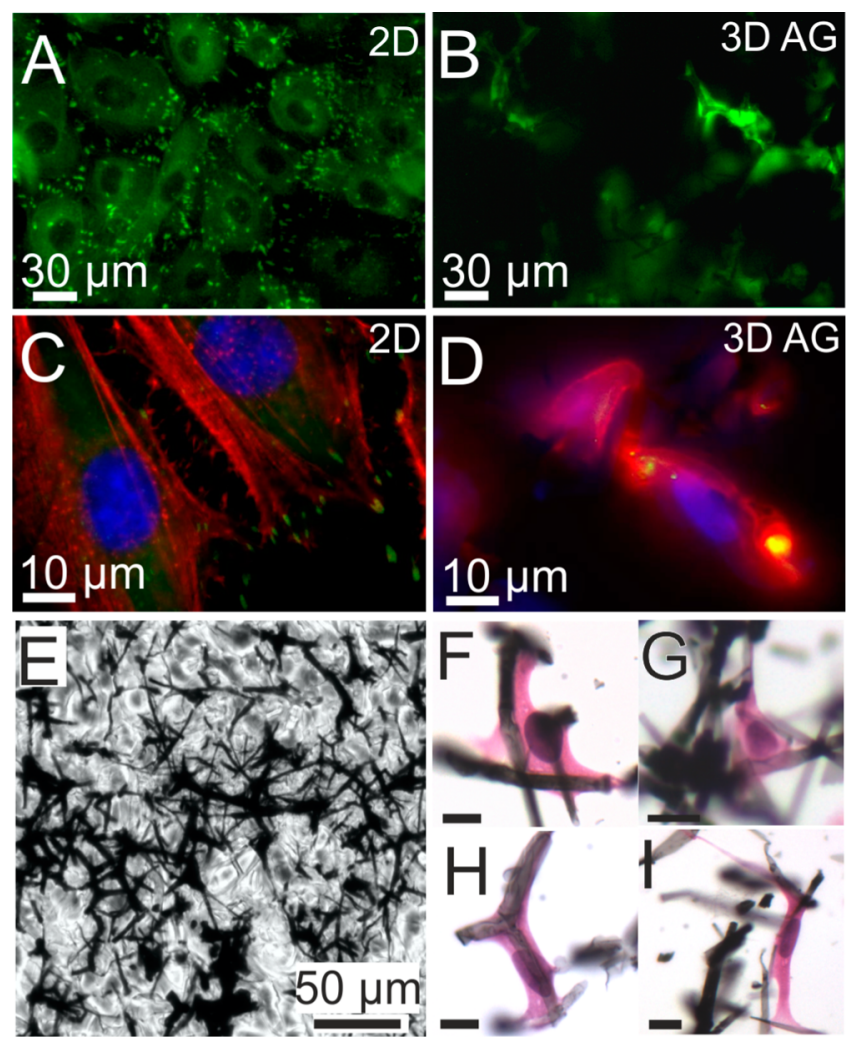

Figure 4. (A) YFP fluorescence (green) images of REF YFP-paxillin cells on a flat substrate, which have grown to a confluent layer. Bright fluorescent spots indicate focal adhesions of the cells. Intracellular homogeneous fluorescence originates in part from cytosolic paxillin. Dark circular regions indicate the area of cell nuclei. B) Optical section image approximately $100 \mu \mathrm{m}$ from the surface. YFP-paxillin fluorescence appears to be associated with filament-like structures indicating cell growth along fibers of the scaffold. (C) Highermagnification fluorescence image of REF YFP-paxillin cells on $2 \mathrm{~d}$ substrate (focal adhesion sites; green) that were stained with DAPI (nuclei; blue) and RFP (stress fibers; red). (D) Optical section image approximately $50 \mu \mathrm{m}$ from the surface, showing a mesh of actin (red) rather than stress fibers and smaller clusters of YFP-paxillin compared to the $2 \mathrm{D}$ substrate, which appear yellow due to overlap with red actin fluorescence. E) Bright field image of a $9 \mu \mathrm{m}$ paraffin thin section from a position about $0.4 \mathrm{~mm}$ below the AG surface. Embedded in wax cells cannot be distinguished from the paraffin background. (F-I) Haematoxylin and eosin staining makes REF52 YFP Pax cells visible by coloring the nuclei blue (hematoxylin) and the cytosol pink (eosin). Due to vigorous dewaxing and staining treatment, the original AG section is highly fragmented. Nevertheless, higher-magnification reveals cells that are well-interfaced with AG filaments and illustrate morphologies typical for fibroblasts. Scale bars: $10 \mu \mathrm{m}$.

$300 \mu \mathrm{m}$ from the surface. Figure 4B shows an optical section image taken approximately $100 \mu \mathrm{m}$ from the surface of an AG scaffold. In the image AG filaments are well visible as black fibers, as they absorbed all emitted light; fluorescence signals from embedded cells indicate progressive growth into the matrix. Figures 4C, D compare actin (red) and paxillin (green) distributions on $2 \mathrm{D}$ glass and in $3 \mathrm{D}$ AG. On a $2 \mathrm{D}$ substrate, actin assembles in stress fibers, and separate focal adhesion sites are clearly visible. In 3D, paxillin clusters are much smaller and actin forms more of a mesh. This is in agreement with previous studies that have shown that adhesion structures are quite different in $2 \mathrm{D}$ and $3 \mathrm{D}$, $^{6,27-29}$
Because of the obvious limitations of fluorescence microscopy due to the optical properties of AG, we prepared histological sections for bright-field microscopy. The samples were dehydrated, embedded in paraffin, and sections of $9 \mu \mathrm{m}$ thickness were cut from the surface down to about half the sample height at $1.5 \mathrm{~mm}$ (see also Figure S4-S6). To visualize the embedded cells, we applied hematoxylin and eosin (HE) stain that color cell nuclei in blue and eosinophilic structures in the cytosol in shades of red and pink. AG scaffold fragments clearly show association with intact cells (Figure 4F-I). Through screening of all sections from multiple scaffolds we determined colonization depths of up to $580 \mu \mathrm{m}$. Highermagnification images revealed fibroblasts of normal morphologies that were well interfaced with AG fragments (Figures $3 \mathrm{~F}-\mathrm{L}$ ) and stretched out or spanned between adjacent filaments, in accordance with our observation from SEM.

In summary, we demonstrated the capacity of biofunctionalized AG as a novel ultra lightweight graphitic material to provide a scaffold conducive to directed three-dimensional cell growth. Cells were able to adhere, extend leading edges and elongate along the fibers of the matrix. The great advantages of AG compared to other porous $3 \mathrm{D}$ scaffolds are its extremely high porosity and the opportunity to tune the elastic modulus to accommodate different types of tissues. ${ }^{20}$ Together with the material's excellent electrical properties (conductivity $\sim 1 \mathrm{~S} / \mathrm{m}$ ) AG may prove very promising for applications where guidance cues and electrical conductivity within a $3 \mathrm{D}$ environment are vital for cell proliferation and stimulation, e.g., in cardiac tissue engineering $^{30,31}$ or regeneration of neural activity. ${ }^{32}$ Thus, our findings commend AG with its extensive possibilities of tailoring for further investigations of other tissue engineering and bioapplications.

\section{ASSOCIATED CONTENT}

\section{S Supporting Information}

The Supporting Information is available free of charge on the ACS Publications website at DOI: 10.1021/acsami.6b00778.

Materials and Methods, test series using different surfactants for AG immersion, additional SEM images of functionalized AG without $\mathrm{Au}$ coating and pristine AG with Au coating, images of paraffin embedded AG, and corresponding thin sections of three types of $A G$ with different specific weight (PDF)

\section{AUTHOR INFORMATION}

\section{Corresponding Author}

*E-mail: constanze.lamprecht@jku.at.

\section{Present Address}

${ }^{\#}$ C.L. is currently at Institute of Biophysics, Johannes Kepler University Linz, 4020 Linz, Austria

\section{Author Contributions}

The manuscript was written through contributions of all authors. All authors have given approval to the final version of the manuscript.

Notes

The authors declare no competing financial interest.

\section{ACKNOWLEDGMENTS}

This project was funded by the European Union's Framework Programme 7 (2007-2013) under the Marie Sklodowska-Curie Grant Agreement 330418. In addition, C.S. acknowledges 
funding from the European Research Council under ERC Starting Grant no. 336104. R.A., I.P., and A.S acknowledge support through German Research Foundation (DFG) grant $\mathrm{AD}$ 183/17-1. M.M. and K.S. received funding through the DFG SFB $986 \mathrm{M}^{3}$ project B1. and M.T. was supported by the Deutscher Akademischer Austauschdienst (DAAD) through a research grant for doctoral candidates (91526555-57048249). We gratefully acknowledge the help of Brook Shurtleff with English editing.

\section{ABBREVIATIONS}

AG, aerographite

CVD, chemical vapor deposition

$\mathrm{CPD}$, super critical point drying

cRGD, cyclic RGD

DSPE-PEG2000- $\mathrm{NH}_{2}$ ，2-distearoyl-sn-glycero-3-phosphoe-

thanolamine-N-[amine(PEG)2000]

ECM, extra cellular matrix

PEG, poly(ethylene glycol)

SEM, scanning electron microscopy

\section{REFERENCES}

(1) Alberts, B.; Johnson, A.; Lewis, J.; Raff, M.; Roberts, K.; Walter, P. The Extracellular Matrix of Animals. In Molecular Biology of the Cell, 4th ed.; Alberts, B, Johnson, A, Lewis, J, Raff, M, Roberts, K., Walter, P.., Eds.; Garland Science: New York, 2002; Chapter 19.

(2) Perez, R. A.; Won, J.-E.; Knowles, J. C.; Kim, H.-W. Naturally and Synthetic Smart Composite Biomaterials for Tissue Regeneration. Adv. Drug Delivery Rev. 2013, 65 (4), 471-496.

(3) Lutolf, M. P.; Hubbell, J. A. Synthetic Biomaterials as Instructive Extracellular Microenvironments for Morphogenesis in Tissue Engineering. Nat. Biotechnol. 2005, 23 (1), 47-55.

(4) Zorlutuna, P.; Annabi, N.; Camci-Unal, G.; Nikkhah, M.; Cha, J. M.; Nichol, J. W.; Manbachi, A.; Bae, H.; Chen, S.; Khademhosseini, A. Microfabricated Biomaterials for Engineering 3D Tissues. Adv. Mater. 2012, 24 (14), 1782-1804.

(5) Bajaj, P.; Schweller, R. M.; Khademhosseini, A.; West, J. L.; Bashir, R. 3D Biofabrication Strategies for Tissue Engineering and Regenerative Medicine. Annu. Rev. Biomed. Eng. 2014, 16, 247-276.

(6) Cukierman, E.; Pankov, R.; Yamada, K. M. Cell Interactions with Three-dimensional Matrices. Curr. Opin. Cell Biol. 2002, 14 (5), 633639.

(7) Grinnell, F. Fibroblast Biology in Three-dimensional Collagen Matrices. Trends Cell Biol. 2003, 13 (5), 264-269.

(8) Oberpenning, F.; Meng, J.; Yoo, J. J.; Atala, A. De Novo Reconstitution of a Functional Mammalian Urinary Bladder by Tissue Engineering. Nat. Biotechnol. 1999, 17 (2), 149-155.

(9) Park, K. I.; Teng, Y. D.; Snyder, E. Y. The injured brain interacts reciprocally with neural stem cells supported by scaffolds to reconstitute lost tissue. Nat. Biotechnol. 2002, 20 (11), 1111-1117.

(10) Yang, S. F.; Leong, K. F.; Du, Z. H.; Chua, C. K. The Design of Scaffolds for Use in Tissue Engineering. Part 1. Traditional Factors. Tissue Eng. 2001, 7 (6), 679-689.

(11) van der Flier, A.; Sonnenberg, A. Function and Interactions of Integrins. Cell Tissue Res. 2001, 305 (3), 285-298.

(12) Hersel, U.; Dahmen, C.; Kessler, H. RGD Modified Polymers: Biomaterials for Stimulated Cell Adhesion and Beyond. Biomaterials 2003, 24 (24), 4385-4415.

(13) Rahmany, M. B.; Van Dyke, M. Biomimetic Approaches to Modulate Cellular Adhesion in Biomaterials: A Review. Acta Biomater. 2013, 9 (3), 5431-5437.

(14) Battista, E.; Causa, F.; Lettera, V.; Panzetta, V.; Guarnieri, D.; Fusco, S.; Gentile, F.; Netti, P. A. Ligand Engagement on Material Surfaces is Discriminated by Cell Mechanosensoring. Biomaterials 2015, 45, 72-80.

(15) Mecklenburg, M.; Schuchardt, A.; Mishra, Y. K.; Kaps, S.; Adelung, R.; Lotnyk, A.; Kienle, L.; Schulte, K. Aerographite: Ultra
Lightweight, Flexible Nanowall, Carbon Microtube Material with Outstanding Mechanical Performance. Adv. Mater. 2012, 24 (26), 3486-3490.

(16) Schuchardt, A.; Braniste, T.; Mishra, Y. K.; Deng, M.; Mecklenburg, M.; Stevens-Kalceff, M. A.; Raevschi, S.; Schulte, K.; Kienle, L.; Adelung, R.; Tiginyanu, I. Three-dimensional AerographiteGaN Hybrid Networks: Single Step Fabrication of Porous and Mechanically Flexible Materials for Multifunctional Applications. Sci. Rep. 2015, 5, 8839.

(17) Crapo, P. M.; Gilbert, T. W.; Badylak, S. F. An Overview of Tissue and Whole Organ Decellularization Processes. Biomaterials 2011, 32 (12), 3233-3243.

(18) Sill, T. J.; von Recum, H. A. Electrospinning: Applications in Drug Delivery and Tissue Engineering. Biomaterials 2008, 29 (13), 1989-2006.

(19) Mishra, Y. K.; Kaps, S.; Schuchardt, A.; Paulowicz, I.; Jin, X.; Gedamu, D.; Freitag, S.; Claus, M.; Wille, S.; Kovalev, A.; Gorb, S. N.; Adelung, R. Fabrication of Macroscopically Flexible and Highly Porous 3D Semiconductor Networks from Interpenetrating Nanostructures by a Simple Flame Transport Approach. Part. Part. Syst. Charact. 2013, 30 (9), 775-783.

(20) Seidi, A.; Ramalingam, M.; Elloumi-Hannachi, I.; Ostrovidov, S.; Khademhosseini, A. Gradient biomaterials for soft-to-hard interface tissue engineering. Acta Biomater. 2011, 7 (4), 1441-51.

(21) Li, N.; Zhang, Q.; Gao, S.; Song, Q.; Huang, R.; Wang, L.; Liu, L.; Dai, J.; Tang, M.; Cheng, G. Three-dimensional Graphene Foam as a Biocompatible and Conductive Scaffold for Neural Stem Cells. Sci. Rep. 2013, 3, 1604.

(22) Serrano, M. C.; Patino, J.; Garcia-Rama, C.; Ferrer, M. L.; Fierro, J. L. G.; Tamayo, A.; Collazos-Castro, J. E.; del Monte, F.; Gutierrez, M. C. 3D Free-standing Porous Scaffolds Made of Graphene Oxide as Substrates for Neural Cell Growth. J. Mater. Chem. B 2014, 2 (34), 5698-5706.

(23) Correa-Duarte, M. A.; Wagner, N.; Rojas-Chapana, J.; Morsczeck, C.; Thie, M.; Giersig, M. Fabrication and Biocompatibility of Carbon Nanotube-based 3D Networks as Scaffolds for Cell Seeding and Growth. Nano Lett. 2004, 4 (11), 2233-2236.

(24) Knop, K.; Hoogenboom, R.; Fischer, D.; Schubert, U. S. Poly(ethylene glycol) in Drug Delivery: Pros and Cons as Well as Potential Alternatives. Angew. Chem., Int. Ed. 2010, 49 (36), 62886308.

(25) Yang, M.; Wada, M.; Zhang, M.; Kostarelos, K.; Yuge, R.; Iijima, S.; Masuda, M.; Yudasaka, M. A High Poly(ethylene glycol) Density on Graphene Nanomaterials Reduces the Detachment of Lipidpoly(ethylene glycol) and Macrophage Uptake. Acta Biomater. 2013, 9 (1), 4744-4753.

(26) Cavalcanti-Adam, E. A.; Volberg, T.; Micoulet, A.; Kessler, H.; Geiger, B.; Spatz, J. P. Cell Spreading and Focal Adhesion Dynamics are Regulated by Spacing of Integrin Ligands. Biophys. J. 2007, 92 (8), 2964-2974.

(27) Baker, B. M.; Chen, C. S. Deconstructing the third dimension how 3D culture microenvironments alter cellular cues. J. Cell Sci. 2012, 125 (13), 3015-3024.

(28) Cukierman, E.; Pankov, R.; Stevens, D. R.; Yamada, K. M. Taking cell-matrix adhesions to the third dimension. Science 2001, 294 (5547), 1708-12.

(29) Kubow, K. E.; Horwitz, A. R. Reducing background fluorescence reveals adhesions in 3D matrices (vol 13, pg 3, 2011). Nat. Cell Biol. 2012, 14 (12), 1344.

(30) Dvir, T.; Timko, B. P.; Brigham, M. D.; Naik, S. R.; Karajanagi, S. S.; Levy, O.; Jin, H. W.; Parker, K. K.; Langer, R.; Kohane, D. S. Nanowired Three-dimensional Cardiac Patches. Nat. Nanotechnol. 2011, 6 (11), 720-725.

(31) Ganji, Y.; Li, Q.; Quabius, E. S.; Böttner, M.; Selhuber-Unkel, C.; Kasra, M. Cardiomyocyte Behavior on Biodegradable Polyurethane/Gold Nanocomposite Scaffolds Under Electrical Stimulation. Mater. Sci. Eng., C 2016, 59, 10-18.

(32) Fabbro, A.; Villari, A.; Laishram, J.; Scaini, D.; Toma, F. M.; Turco, A.; Prato, M.; Ballerini, L. Spinal Cord Explants Use Carbon 
Nanotube Interfaces To Enhance Neurite Outgrowth and To Fortify Synaptic Inputs. ACS Nano 2012, 6 (3), 2041-2055. 